\title{
Dynamic kinetic resolution of $(S)$-mandelate-derived $\alpha$-bromo esters in nucleophilic substitution and asymmetric syntheses of 3-substituted morpholin-2-ones
}

\author{
Yoon Min Lee, Kyoung Hee Kang, Hye-Min Min, Hyun Jin Lim, Eun-Hyung Park, \\ and Yong Sun Park* \\ Department of Chemistry, Konkuk University, Seoul 143-701, Korea \\ E-mail:parkyong@konkuk.ac.kr
}

\begin{abstract}
Dynamic kinetic resolution of $(S)$-mandelate-derived $\alpha$-bromo esters in nucleophilic substitution reaction has been investigated. Substitutions with various alkyl amine nucleophiles in the presence of TBAI and DIEA can provide various $\alpha$-amino esters up to $81 \%$ yield and 97:3 dr. Also, the substitution of $\alpha$-bromo esters with $N$-substituted 2-aminoethanol nucleophiles and following spontaneous cyclization provides a practical protocol for asymmetric syntheses of 3substituted morpholin-2-ones up to 95:5 er.
\end{abstract}

Keywords: Chiral auxiliary, dynamic kinetic resolution, nucleophilic substitution, asymmetric synthesis, morpholinone

\section{Introduction}

Dynamic kinetic resolution of chiral alcohol-derived $\alpha$-halo esters has been recently recognized as an effective synthetic method for asymmetric syntheses of $\alpha$-heteroatom substituted carboxylic acid derivatives. $^{1-3}$ While the chiral auxiliaries can achieve a useful level of stereoselectivity, it is still desirable to find novel ways to utilize the methodology for practical asymmetric syntheses. We have previously reported $(S)$-mandelate-mediated dynamic kinetic resolution of $\alpha$-bromo esters with various aryl amines for asymmetric syntheses of dihydroquinoxalinones and dihydrobenzoxazinones. ${ }^{4}$ Herein we report our recent results on the asymmetric nucleophilic substitution of (S)-mandelate-derived $\alpha$-bromo esters with various alkyl amines for practical asymmetric syntheses of morpholin-2-ones. 


\section{Results and Discussion}

Initial studies on $(S)$-mandelate-mediated dynamic kinetic resolution were carried out with $\alpha$ bromo phenylacetate 1 and dibenzylamine $\left(\mathrm{Bn}_{2} \mathrm{NH}\right)$. When the diastereomeric mixture (1:1) of $(\alpha R S)-1$ was treated with tetrabutylammonium iodide (TBAI, 1.0 equiv), diisopropylethylamine (DIEA, 1.0 equiv) and dibenzylamine (1.5 equiv) in $\mathrm{CH}_{2} \mathrm{Cl}_{2}$ at room temperature for $12 \mathrm{~h}$, the amino acid derivative $(\alpha R)-2$ was produced in $81 \%$ yield with 97:3 diastereomeric ratio (dr) as shown in Scheme 1. Subsequent reductive cleavage of $(\alpha R)-2$ using $\mathrm{LiAlH}_{4}$ furnished the enantioenriched $N, N$-dibenzyl 2-aminoalcohol $(R)-3$ in 86\% yield with 97:3 enantiomeric ratio (er). The results imply that the $\alpha$-bromo stereogenic center is configurationally labile with respect to the rate of substitution and $(\alpha R S)-\mathbf{1}$ is dynamically resolved under the reaction condition.

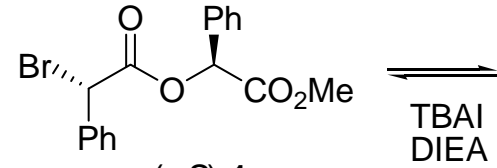

$(\alpha S)-1$

$$
\mathrm{Bn}_{2} \mathrm{NH}
$$<smiles>CC(=O)C(OC(=O)[C@@H](Nc1ccccc1)c1ccccc1)c1ccccc1</smiles>

$(\alpha R)-2$

$97: 3 \mathrm{dr}$<smiles>CC(=O)C(OC(=O)C(Br)c1ccccc1)c1ccccc1</smiles>

$(\alpha R)-1$
$\mathrm{LiAlH}_{4}$<smiles>OCC(Nc1ccccc1)c1ccccc1</smiles>

$(R)-3$

$97: 3$ er

Scheme 1. Dynamic kinetic resolution in nucleophilic substitution.

The scope of the observed dynamic kinetic resolution has been examined with $\alpha$-bromo propionate $\mathbf{4}$ as shown in Table 1. When the diastereomeric mixture (1:1) of $(\alpha R S)-\mathbf{4}$ was treated with dibenzylamine (1.5 equiv), TBAI (1.0 equiv) and DIEA (1.0 equiv) for $24 \mathrm{~h}$, the amino ester $(\alpha R)$-11 was obtained in 62\% yield with 90:10 dr. (entry 1) In the absence of DIEA, the rate of the substitution was substantially decreased to provide $\mathbf{1 1}$ in 38\% yield after $24 \mathrm{~h}$ with the same selectivity (entry 2). The reaction of $\mathbf{4}$ in the absence of TBAI, however, gave $\mathbf{1 1}$ with a lower stereoselectivity. (entry 3) The substitution of $\mathbf{4}$ was very slow in the absence of both TBAI and DIEA to provide 11 in 19\% yield with 86:14 dr. (entry 4). The results in entries 2-4 pointed to the importance of the presence of halide ion and base for sufficient rate acceleration and selectivity. The lack of an extraneous halide ion would inhibit the rapid epimerization of 4 thereby decreasing the stereoselection. As shown in entries 5-9, most of the solvents explored 
gave similar selectivities to give 11 with 90:10 dr in $\mathrm{CH}_{3} \mathrm{CN}, 87: 13 \mathrm{dr}$ in THF, 89:11 $\mathrm{dr}$ in dioxane, 87:13 $\mathrm{dr}$ in acetone and 88:12 $\mathrm{dr}$ in ethyl acetate. However, the selectivity was reduced in DMSO or in DMF (entries 10-11) and the reaction was very slow in $n$-hexane or in diethyl ether. Decreasing the reaction temperature reduced the rate of the reaction significantly and a slightly lower selectivity $(88: 12 \mathrm{dr})$ of $\mathbf{1 1}$ was observed at $0^{\circ} \mathrm{C}$.

Table 1. Substitutions under various reaction conditions

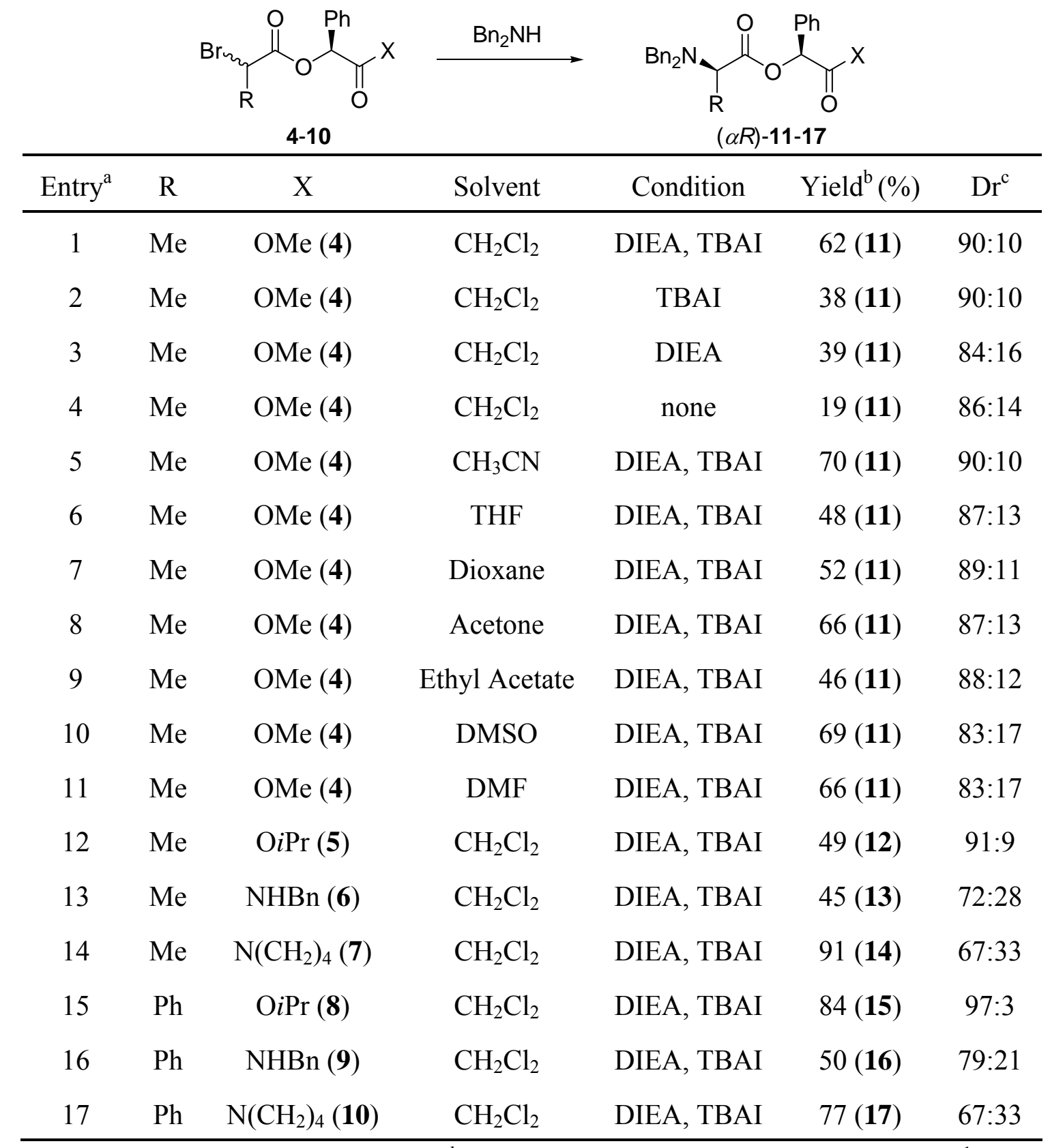

${ }^{\mathrm{a}}$ The reactions were carried out for $24 \mathrm{~h} .{ }^{\mathrm{b}}$ Isolated yields. ${ }^{\mathrm{c}}$ The drs are determined by ${ }^{1} \mathrm{H}$ NMR of reaction mixture. 
In an effort to improve the stereoselectivity, we examined the substitutions of $\alpha$-bromo propionates with three different chiral auxiliaries derived from (S)-mandelic acid. No substantial difference has been found in the reaction of $\mathbf{5}$ derived from isopropyl mandelate. However, much lower stereoselectivities were noted for the substitution reactions of $\mathbf{6}$ and $\mathbf{7}$ derived from mandelamides. (entries 12-14) Also, a similar trend in stereoselectivity was observed with $\alpha$ bromo phenylacetates $\mathbf{8}, \mathbf{9}$ and 10. (entries 15-17)

Table 2. Substitutions with various alkyl amine nucleophiles

$$
\begin{aligned}
& \mathbf{1}(\mathrm{R}=\mathrm{Ph}) \\
& \mathbf{4}(\mathrm{R}=\mathrm{Me}) \\
& \mathbf{1 8}(\mathrm{R}=\mathrm{Et}) \\
& \mathbf{1 9}(\mathrm{R}=n-\mathrm{Bu})
\end{aligned}
$$

\begin{tabular}{|c|c|c|c|c|}
\hline Entry $^{\mathrm{a}}$ & Nucleophile & $\mathrm{R}$ & Yield $^{\mathrm{b}}(\%)$ & $\mathrm{Dr}^{\mathrm{c}}$ \\
\hline 1 & & $\mathrm{Ph}$ & $92(\mathbf{2 0})$ & 91:9 \\
\hline 2 & & $\mathrm{Me}$ & $88(21)$ & $88: 12$ \\
\hline 3 & & $\mathrm{Ph}$ & 86 (22) & $90: 10$ \\
\hline 4 & & $\mathrm{Me}$ & $82(23)$ & $88: 12$ \\
\hline 5 & & $\mathrm{Me}$ & 75 (24) & $68: 32$ \\
\hline 6 & & Et & $82(25)$ & $72: 28$ \\
\hline 7 & & $\mathrm{Bu}$ & $69(\mathbf{2 6})$ & $75: 25$ \\
\hline 8 & & $\mathrm{Ph}$ & 87 (27) & $82: 18$ \\
\hline 9 & & $\mathrm{Me}$ & $92(\mathbf{2 8})$ & $78: 22$ \\
\hline 10 & & $\mathrm{Ph}$ & $43(29)$ & $93: 7$ \\
\hline 11 & & $\mathrm{Me}$ & $72(30)$ & $78: 22$ \\
\hline 12 & & $\mathrm{Ph}$ & $88(\mathbf{3 1})$ & $88: 12$ \\
\hline 13 & & Et & $79(32)$ & $78: 22$ \\
\hline
\end{tabular}<smiles>[R]C(C#N)C(=O)OC(C(C)=O)c1ccccc1</smiles>

$(\alpha R)-20-32$

${ }^{a}$ The reactions were carried out for $24 \mathrm{~h} .{ }^{\mathrm{b}}$ Isolated yields. ${ }^{\mathrm{c}}$ The drs are determined by ${ }^{1} \mathrm{H}$ NMR of reaction mixture.

Next, we examined six different alkyl amine nucleophiles to evaluate the scope of the dynamic kinetic resolution as shown in Table 2 . The treatment of $\alpha$-bromo phenylacetate $\mathbf{1}$ with 
dibutylamine (1.5 equiv) in $\mathrm{CH}_{2} \mathrm{Cl}_{2}$ for $24 \mathrm{~h}$ at room temperature gave 20 in $92 \%$ yield with 91:9 dr. (entry 1) Under the same reaction conditions, the reaction of $\alpha$-bromo propionate 4 with the secondary amine gave $N, N$-dibutyl amino ester 21 with a slightly lower dr. (entry 2) In the reactions with diallylamine nucleophile, similar stereoselectivities were obtained in the reactions of $\alpha$-bromo acetates 1 and 4. (entries 3-4) Notably, when cyclic secondary amine nucleophiles were used, the reactions gave much lower stereoselectivities (entries 5-9). Also, the reactions with two primary amines provided the products 29-32 with lower selectivities compared to the reactions of the corresponding $\alpha$-bromo acetates with dibenzylamine (entries 10-13). Limited results in Table 2 indicate that both the size of amine nucleophiles and the nature of the $\alpha$ substituent of $\alpha$-bromo acetates significantly affect the stereoselectivity of the nucleophilic substitution.

Table 3. Asymmetric synthesis of 3-substituted morpholin-2-one

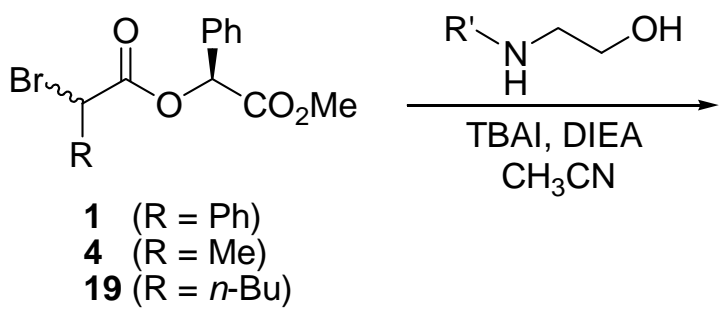<smiles>[R]C1C(=O)OCCN1[R]</smiles>

$(R)-33-39$

\begin{tabular}{|c|c|c|c|c|}
\hline Entry $^{\mathrm{a}}$ & $\mathrm{R}$ & $\mathrm{R}$ & Yield $^{\mathrm{b}}(\%)$ & $\mathrm{Er}^{\mathrm{c}}$ \\
\hline 1 & $\mathrm{Ph}$ & & 81 (33) & $90: 10$ \\
\hline 2 & $\mathrm{Me}$ & & 77 (34) & $84: 16$ \\
\hline 3 & $n-\mathrm{Bu}$ & & 72 (35) & $67: 33$ \\
\hline 4 & $\mathrm{Ph}$ & & $60(36)$ & $87: 13$ \\
\hline 5 & $\mathrm{Ph}$ & & $58(37)$ & 91:9 \\
\hline
\end{tabular}




\begin{tabular}{ccccc}
\hline Entry $^{\mathrm{a}}$ & $\mathrm{R}$ & $\mathrm{R}$ & Yield $^{\mathrm{b}}(\%)$ & $\mathrm{Er}^{\mathrm{c}}$ \\
\hline 6 & $\mathrm{Ph}$ & $55(38)$ & $91: 9$ \\
7 & $\mathrm{Ph}$ & & \\
\hline
\end{tabular}

${ }^{a}$ The reactions were carried out for $24-72 \mathrm{~h} .{ }^{\mathrm{b}}$ Isolated yields. ${ }^{\mathrm{c}}$ The ers are determined by chiral stationary phase-HPLC.

Encouraged by the high enantioselectivities in the reactions of $\alpha$-bromo acetates with various secondary amines, we set out to examine the substitutions with $\mathrm{N}$-substituted 2-aminoethanol nucleophiles for asymmetric syntheses of 3-substituted morpholin-2-ones as shown in Table 3. When $\alpha$-bromo phenylacetate 1 was treated with $N$-benzyl 2 -aminoethanol, TBAI and DIEA in $\mathrm{CH}_{3} \mathrm{CN}$ for $24 \mathrm{~h}$ at room temperature, we were pleased to observe that the substitution and following spontaneous cyclization gave $N$-benzyl 3-phenyl-morpholin-2-one 33 in $81 \%$ yield with 90:10 $\mathrm{er}^{5}$ (entry 1). The reactions of $\alpha$-bromo acetates 4 and 19 the reactions in $\mathrm{CH}_{3} \mathrm{CN}$ afforded 3-alkyl substituted morpholin-2-ones $\mathbf{3 4}$ and $\mathbf{3 5}$ with lower stereoselectivities of 84:16 er and 67:33 er, respectively (entries 2-3). In an effort to improve the stereoselectivity, we tested various 2-aminoethanol nucleophiles with different $N$-substituents. The reactions of $\alpha$-bromo phenylacetate 1 with three different $N$-alkyl 2-aminoethanol nucleophiles produced morpholin-2ones 36-38 with similar yields and enantioselectivities. (entries 4-6) As with $N$ - $p$ methoxyphenyl 2-aminoethanol nucleophile, the reaction of $\alpha$-bromo acetate 1 provided 3phenyl-morpholin-2-one 39 with higher stereoselectivity (95:5 dr) compared to the reaction with $\mathrm{N}$-benzyl 2-aminoethanol. (entry 7) The spontaneous cyclization in the reaction with $\mathrm{N}$-aryl substituted 2-aminoethanol was relatively slower and $\mathrm{N}$-aryl morpholin-2-one 39 was obtained in $66 \%$ yield after $3 \mathrm{~d}$ stirring. 

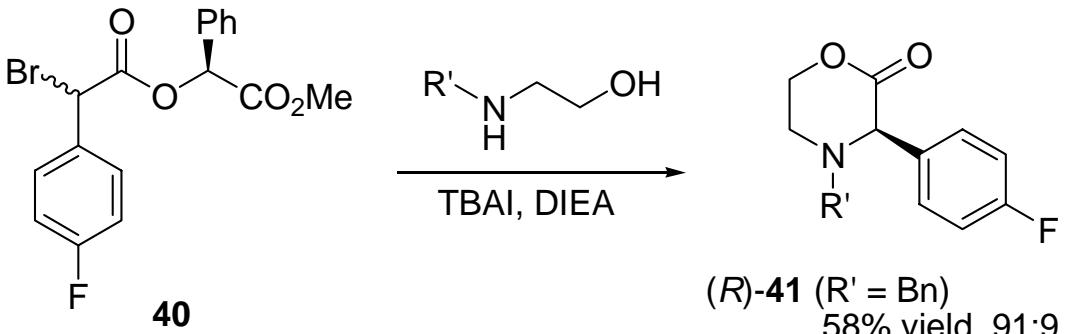

$(R)-41\left(\mathrm{R}^{\prime}=\mathrm{Bn}\right)$

$58 \%$ yield, $91: 9$ er

$(R)-42\left(\mathrm{R}^{\prime}=p-\mathrm{MeO}-\mathrm{Ph}\right)$

$72 \%$ yield, $94: 6$ er

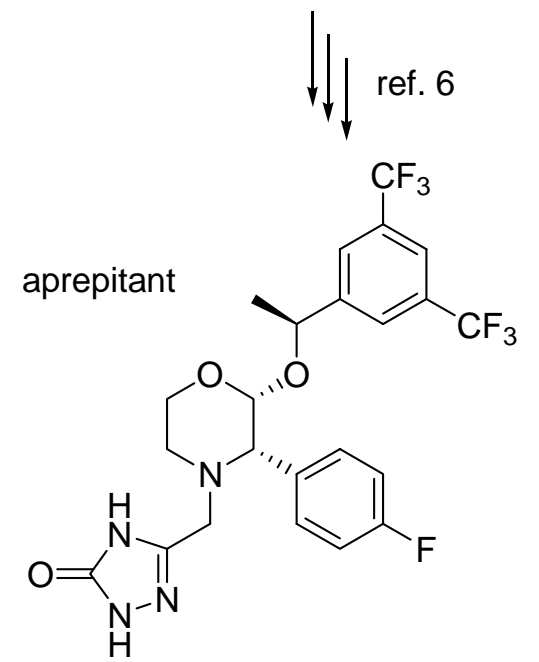

Scheme 2. Asymmetric synthesis of key intermediates of aprepitant.

As an application of the methodology we demonstrated the practical asymmetric syntheses of 3-(4-fluorophenyl)-morpholin-2-ones 41 and 42, the key intermediates in the synthesis of aprepitant known as a potent $\mathrm{NK}_{1}$ receptor antagonist. ${ }^{6}$ When $\alpha$-bromo acetate $\mathbf{4 0}$ was treated with $\mathrm{N}$-substituted 2-aminoethanol in the presence of TBAI and DIEA in $\mathrm{CH}_{3} \mathrm{CN}$, the substitution and spontaneous cyclization gave 3-(4-fluorophenyl)-morpholin-2-ones 41 and 42 with 91:9 er and 94:6 er, respectively as shown in Scheme 2.

\section{Conclusions}

We conclude that dynamic kinetic resolution of $(S)$-mandelate-derived $\alpha$-bromo esters in nucleophilic substitution with alkyl amines can be successfully applied towards the preparation of various enantioenriched amino acid derivatives. The results showed that stereoselectivity depends critically on the $\alpha$-substituent of the $\alpha$-bromo acetate and the structure of the amine nucleophiles. The substitution with $N$-substituted 2-aminoethanol nucleophiles and subsequent spontaneous cyclization can provide a general procedure for asymmetric syntheses of 3- 
substituted $(R)$-morpholin-2-ones. The mild and practical synthetic method for optically active amino acid derivatives suggests that this DKR approach should be further developed.

\section{Experimental Section}

General. All reactions were performed in oven-dried glassware under nitrogen atmosphere with freshly distilled solvents. Analytical thin layer chromatography (TLC) was performed on silica gel plates with QF-254 indicator and TLC visualization was carried out with UV-light. Flash column chromatography was performed with 230-400 mesh silica gel. Analytical chiral stationary phase HPLC was performed on pump system coupled to absorbance detector (254nm). Chiral stationary phase columns $(25 \mathrm{~cm} \times 4.6 \mathrm{~mm}$ i.d. $)$ with isopropanol/hexane mobile phase were used to determine enantiomeric ratios. ${ }^{1} \mathrm{H}$ and ${ }^{13} \mathrm{C}$ NMR spectra were acquired on U400 $\left(400 \mathrm{MHz}{ }^{1} \mathrm{H}, 100.6 \mathrm{MHz}{ }^{13} \mathrm{C}\right)$ spectrometer using chloroform-d $\left(\mathrm{CDCl}_{3}\right)$ and purity of the compounds was established to be $>95 \%$ based on ${ }^{1} \mathrm{H}$ and ${ }^{13} \mathrm{C}$ NMR spectra.

General procedure for the preparation of $\alpha$-halo esters $1,4-10,18,19$ and 40. Mandelic acid derived ester or amide (1.0 equiv), racemic $\alpha$-bromo acid (1.0 equiv), DCC (1.0 equiv) and DMAP (0.2 equiv) were dissolved in $\mathrm{CH}_{2} \mathrm{Cl}_{2}$ and stirred at room temperature for 3-10 h. The precipitate was filtered off and the organic phase was washed with water. The organic phase was dried over $\mathrm{MgSO}_{4}$, filtered and concentrated to provide the crude product that was purified by column chromatography on silica gel.

(2-Methoxy-2-oxo-(S)-1-phenylethyl) $\alpha$-bromo-phenylacetate 1. A pale yellow oil was obtained in $54 \%$ yield as a mixture of two diastereomers. ${ }^{1} \mathrm{H} \mathrm{NMR}\left(\mathrm{CDCl}_{3}, 400 \mathrm{MHz}\right.$, two diastereomers) 7.57-7.32 (m, 5H), 5.98, $5.97(\mathrm{~s}, 1 \mathrm{H}), 5.51,5.49(\mathrm{~s}, 1 \mathrm{H}), 3.69,3.63(\mathrm{~s}, 3 \mathrm{H}) ;{ }^{13} \mathrm{C}$ NMR $\left(\mathrm{CDCl}_{3}, 100 \mathrm{MHz}\right)$ 168.9, 168.1, 135.8, 133.4, 129.9, 129.8, 129.3, 129.2, 128.0, 76.2, 53.2, 46.5; Anal. calcd for $\mathrm{C}_{17} \mathrm{H}_{15} \mathrm{BrO}_{4}$ : C, 56.22; H, 4.16; Found: C, 56.26; H, 4.35 .

(2-Methoxy-2-oxo-(S)-1-phenylethyl) $\alpha$-bromo-propanoate 4. A pale yellow oil was obtained in $43 \%$ yield as a mixture of two diastereomers. ${ }^{1} \mathrm{H} \mathrm{NMR}\left(\mathrm{CDCl}_{3}, 400 \mathrm{MHz}\right.$, two diastereomers) 7.48-7.38 (m, 5H), 5.97, 5.96 (s, 1H), 4.52, 4.47 (q, $J=6.9 \mathrm{~Hz}, 1 \mathrm{H}), 3.70$ (s, 3H), 1.87 (d, $J=7.0$ $\mathrm{Hz}, 3 \mathrm{H}) ;{ }^{13} \mathrm{C} \mathrm{NMR}\left(\mathrm{CDCl}_{3}, 100 \mathrm{MHz}\right) 170.0,169.1,133.5,129.9,129.3,128.0,75.8,53.1,39.8$, 22.0; Anal. calcd for $\mathrm{C}_{12} \mathrm{H}_{13} \mathrm{BrO}_{4}$ : C, 47.86; H, 4.35; Found: C, 47.84; H, 4.45.

(2-Isopropoxy-2-oxo-(S)-1-phenylethyl) $\alpha$-bromo-propanoate 5 . A pale yellow oil was obtained in $61 \%$ yield as a mixture of two diastereomers. ${ }^{1} \mathrm{H}$ NMR $\left(\mathrm{CDCl}_{3}, 400 \mathrm{MHz}\right.$, two diastereomers) 7.50-7.39 (m, 5H), 5.90, (s, 1H), $5.06(\mathrm{~m}, 1 \mathrm{H}), 4.53,4.48(\mathrm{q}, J=6.9 \mathrm{~Hz}, 1 \mathrm{H})$, $1.89(\mathrm{~d}, J=7.0 \mathrm{~Hz}, 3 \mathrm{H}), 1.27(\mathrm{~d}, J=6.3 \mathrm{~Hz}, 3 \mathrm{H}), 1.14(\mathrm{~m}, 3 \mathrm{H}) ;{ }^{13} \mathrm{C} \mathrm{NMR}\left(\mathrm{CDCl}_{3}, 100 \mathrm{MHz}\right)$ 169.9, 168.1, 133.8, 129.7, 129.2, 127.9, 76.1, 70.2, 39.7, 22.1; HRMS calcd for $\mathrm{C}_{14} \mathrm{H}_{18} \mathrm{BrO}_{4}$ $\left(\mathrm{M}^{+}+1\right): 329.0388$. Found: 329.0388 .

(2-Benzylamino-2-oxo-(S)-1-phenylethyl) $\alpha$-bromo-propanoate 6. A pale yellow oil was obtained in $31 \%$ yield as a mixture of two diastereomers. ${ }^{1} \mathrm{H} \mathrm{NMR}\left(\mathrm{CDCl}_{3}, 400 \mathrm{MHz}\right.$, two 
diastereomers) 7.47-7.20 (m, 10H), 6.74, $6.61(\mathrm{br}, 1 \mathrm{H}), 6.20,6.12(\mathrm{~s}, 1 \mathrm{H}), 4.45(\mathrm{~m}, 3 \mathrm{H}), 1.81(\mathrm{~d}$, $J=6.9 \mathrm{~Hz}, 3 \mathrm{H}) ;{ }^{13} \mathrm{C} \mathrm{NMR}\left(\mathrm{CDCl}_{3}, 100 \mathrm{MHz}\right) 168.2,137.9,135.1,129.6,129.3,128.2,128.1$, 127.9, 127.7, 76.9, 43.9, 40.3, 22.0; $\mathrm{C}_{18} \mathrm{H}_{18} \mathrm{BrNO}_{3}$ : C, 57.46; H, 4.82; N, 3.72; Found: C, 57.35; H, 4.93; N, 3.89 .

(2-Oxo-2-(1-pyrrolidinyl)-(S)-1-phenylethyl) $\alpha$-bromo-propanoate 7. A pale yellow oil was obtained in $38 \%$ yield as a mixture of two diastereomers. ${ }^{1} \mathrm{H} \mathrm{NMR}\left(\mathrm{CDCl}_{3}, 400 \mathrm{MHz}\right.$, two diastereomers) 7.51-7.29 (m, 5H), 6.05, $6.04(\mathrm{~s}, 1 \mathrm{H}), 4.50(\mathrm{~m}, 1 \mathrm{H}), 3.59(\mathrm{~m}, 2 \mathrm{H}), 3.41(\mathrm{~m}, 1 \mathrm{H})$, $3.14(\mathrm{~m}, 1 \mathrm{H}), 1.94-1.75(\mathrm{~m}, 7 \mathrm{H}) ;{ }^{13} \mathrm{C} \mathrm{NMR}\left(\mathrm{CDCl}_{3}, 100 \mathrm{MHz}\right) 170.5,166.1,133.7,129.9,129.4$, 129.0, 76.0, 46.7, 46.3, 40.5, 26.5, 24.2, 22.2; Anal. calcd for $\mathrm{C}_{15} \mathrm{H}_{18} \mathrm{BrNO}_{3}$ : C, 52.96; H, 5.33; N, 4.12; Found: C, 52.83; H, 5.44; N, 4.13.

(2-Isopropoxy-2-oxo-(S)-1-phenylethyl) $\alpha$-bromo-phenylacetate 8. A pale yellow oil was obtained in $71 \%$ yield as a mixture of two diastereomers. ${ }^{1} \mathrm{H} \mathrm{NMR}\left(\mathrm{CDCl}_{3}, 400 \mathrm{MHz}\right.$, two diastereomers) 7.57-7.30 (m, 10H), 5.92, $5.90(\mathrm{~s}, 1 \mathrm{H}), 5.49,5.47(\mathrm{~s}, 1 \mathrm{H}), 5.06-4.96(\mathrm{~m}, 1 \mathrm{H})$, 1.23, 1.17, 1.10, $1.03(\mathrm{~d}, J=6.3 \mathrm{~Hz}, 6 \mathrm{H}) ;{ }^{13} \mathrm{C} \mathrm{NMR}\left(\mathrm{CDCl}_{3}, 100 \mathrm{MHz}\right) 168.1,167.9,153.8$, 133.7, 133.6, 129.8, 129.4, 129.3, 129.2, 127.9, 76.7, 70.1, 46.7, 22.0, 21.8; Anal. calcd for $\mathrm{C}_{19} \mathrm{H}_{19} \mathrm{BrO}_{4}$ : C, 58.33; H, 4.89; Found: C, 58.22; H, 4.96.

(2-Benzylamino-2-oxo-(S)-1-phenylethyl) $\alpha$-bromo-phenylacetate 9. A pale yellow oil was obtained in $40 \%$ yield as a mixture of two diastereomers. ${ }^{1} \mathrm{H} \mathrm{NMR}\left(\mathrm{CDCl}_{3}, 400 \mathrm{MHz}\right.$, two diastereomers) 7.38-7.05 (m, 15H), 6.52, $6.36(\mathrm{br}, 1 \mathrm{H}), 6.17,6.15(\mathrm{~s}, 1 \mathrm{H}), 5,45,5.42(\mathrm{~s}, 1 \mathrm{H})$, 4.43-4.32 (m, 2H); ${ }^{13} \mathrm{C} \mathrm{NMR}\left(\mathrm{CDCl}_{3}, 100 \mathrm{MHz}\right)$ 168.0, 166.8, 137.8, 135.0, 130.0, 129.7, 129.5, 129.3, 129.2, 128.9, 128.2, 128.1, 127.8, 127.7, 77.2, 47.4, 43.8; Anal. calcd for $\mathrm{C}_{23} \mathrm{H}_{20} \mathrm{BrNO}_{3}$ : C, 63.02; H, 4.60; N, 3.20; Found: C, 63.08; H, 4.70; N, 3.23.

(2-Oxo-2-(1-pyrrolidinyl)-(S)-1-phenylethyl) $\alpha$-bromo-phenylacetate 10. A pale yellow oil was obtained in $32 \%$ yield as a mixture of two diastereomers. ${ }^{1} \mathrm{H}$ NMR $\left(\mathrm{CDCl}_{3}, 400 \mathrm{MHz}\right.$, two diastereomers) 7.56-7.28 (m, 10H), 6.08, $6.06(\mathrm{~s}, 1 \mathrm{H}), 5.51,5.50(\mathrm{~s}, 1 \mathrm{H}), 3.59(\mathrm{~m}, 2 \mathrm{H}), 3.39(\mathrm{~m}$, 1H), $3.14(\mathrm{~m}, 1 \mathrm{H}), 1.93-1.73(\mathrm{~m}, 4 \mathrm{H}) ;{ }^{13} \mathrm{C} \mathrm{NMR}\left(\mathrm{CDCl}_{3}, 100 \mathrm{MHz}\right) 168.6,165.9,136.1,133.6$, $129.9,129.4,129.3,129.2,129.0,128.4,76.3,46.8,46.7,46.4,26.5,24.2$. The spectral data of 10 were identical to those of the authentic material reported previously. ${ }^{\text {le }}$

(2-Methoxy-2-oxo-(S)-1-phenylethyl) $\alpha$-bromo-butanoate 18. A pale yellow oil was obtained in $77 \%$ yield as a mixture of two diastereomers. ${ }^{1} \mathrm{H} \mathrm{NMR}\left(\mathrm{CDCl}_{3}, 400 \mathrm{MHz}\right.$, two diastereomers $)$ 7.48-7.38 (m, 5H), $5.97(\mathrm{~s}, 1 \mathrm{H}), 4.33,4.28(\mathrm{t}, J=7.5$ and $6.8 \mathrm{~Hz}, 1 \mathrm{H}), 3.72(\mathrm{~s}, 3 \mathrm{H}), 2.23 \sim 2.03$ $(\mathrm{m}, 2 \mathrm{H}), 1.08,1.06(\mathrm{t}, J=7.4 \mathrm{~Hz}, 3 \mathrm{H})$. The spectral data of 18 were identical to those of the authentic material reported previously. ${ }^{1 \mathrm{e}}$

(2-Methoxy-2-oxo-(S)-1-phenylethyl) $\alpha$-bromo-haxanoate 19. A pale yellow oil was obtained in $47 \%$ yield as a mixture of two diastereomers. ${ }^{1} \mathrm{H} \mathrm{NMR}\left(\mathrm{CDCl}_{3}, 400 \mathrm{MHz}\right.$, two diastereomers $)$ 7.47-7.35 (m, 5H), $5.96(\mathrm{~s}, 1 \mathrm{H}), 4.37,4.32(\mathrm{t}, J=7.6$ and $7.2 \mathrm{~Hz}, 1 \mathrm{H}), 3.66(\mathrm{~s}, 3 \mathrm{H}), 2.17(\mathrm{~m}$,

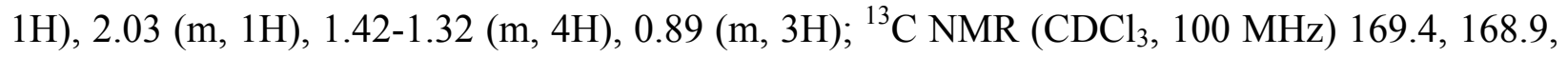
133.6, 129.7, 129.2, 128.0, 75.3, 52.8, 45.7, 35.0, 29.6, 21.4, 14.2; Anal. calcd for $\mathrm{C}_{15} \mathrm{H}_{19} \mathrm{BrO}_{4}$ : C, 52.49; H, 5.58; Found: C, 52.54; H, 5.70. 
(2-Methoxy-2-oxo-(S)-1-phenylethyl) $\alpha$-bromo-(p-fluorophenyl)acetate 40. A pale yellow oil was obtained in $77 \%$ yield as a mixture of two diastereomers. ${ }^{1} \mathrm{H}$ NMR $\left(\mathrm{CDCl}_{3}, 400 \mathrm{MHz}\right.$, two diastereomers) 7.57-7.35 (m, 5H), $7.03(\mathrm{~m}, 2 \mathrm{H}), 5.98,5.97(\mathrm{~s}, 1 \mathrm{H}), 5.50,5.47(\mathrm{~s}, 1 \mathrm{H}), 3.70,3.65$ $(\mathrm{s}, 3 \mathrm{H}) ;{ }^{13} \mathrm{C} \mathrm{NMR}\left(\mathrm{CDCl}_{3}, 100 \mathrm{MHz}\right) 168.8,167.9,163.5(\mathrm{~d}, J=247.9 \mathrm{~Hz}, 1 \mathrm{H}), 133.4,131.7$, 131.6, $131.3(\mathrm{~d}, J=8.5 \mathrm{~Hz}, 1 \mathrm{H}), 130.0,129.3,128.0,116.3(\mathrm{~d}, J=21.6 \mathrm{~Hz}, 1 \mathrm{H}), 76.4,53.2$, 45.5; Anal. calcd for $\mathrm{C}_{17} \mathrm{H}_{14} \mathrm{BrFO}_{4}$ : C, 53.56; H, 3.70; Found: C, 53.44; H, 3.57.

General procedure for the asymmetric preparation of 2, 11-17, 20-39 and 41-42. To a solution of $\alpha$-bromo ester $\left(1,4-10,18,19\right.$ and 40 ) in $\mathrm{CH}_{2} \mathrm{Cl}_{2}$ or $\mathrm{CH}_{3} \mathrm{CN}$ (ca. $0.1 \mathrm{M}$ ) at room temperature were added DIEA (1.0 equiv), TBAI (1.0 equiv) and a nucleophile (1.5 equiv). After the resulting reaction mixture was stirred at room temperature for $12-72 \mathrm{~h}$, the solvent was evaporated and the crude material was purified by column chromatography to give a $\alpha$-amino ester. The drs of 2, 11-17, 20-32 were determined by ${ }^{1} \mathrm{H}$ NMR integration of $\alpha$-hydrogens of two diastereomers and the ers of 3, 33-39 and 41-42 were determined by chiral stationary phase HPLC.

(2-Methoxy-2-oxo-(S)-1-phenylethyl) $\quad(\boldsymbol{R})$ - $\alpha$-dibenzylamino-phenylacetate $\quad 2 . \quad{ }^{1} \mathrm{H} \quad \mathrm{NMR}$ $\left(\mathrm{CDCl}_{3}, 400 \mathrm{MHz}\right.$, major diastereomer) 7.43-7.22(m, 20H), $6.05(\mathrm{~s}, 1 \mathrm{H}), 4.81(\mathrm{~s}, 1 \mathrm{H}), 3.83$ (s, 4H), $3.79(\mathrm{~s}, 3 \mathrm{H}) ;{ }^{13} \mathrm{C} \mathrm{NMR}\left(\mathrm{CDCl}_{3}, 100 \mathrm{MHz}\right) 172.2,169.7,140.0,136.7,133.9,129.7,129.4$, $129.2,128.8,128.7,128.3,128.1,127.5,75.1,65.8,54.5,53.2$; Anal. calcd for $\mathrm{C}_{31} \mathrm{H}_{29} \mathrm{NO}_{4}$ : C, 77.64; H, 6.10; N, 2.92; Found: C, 77.20; H, 6.18; N, 2.84.

(R)-2-dibenzylamino-2-phenylethanol 3. ${ }^{1} \mathrm{H}$ NMR $\left(\mathrm{CDCl}_{3}, 400 \mathrm{MHz}\right)$ 7.44-7.25 (m, 15H), 4.14 $(\mathrm{dd}, J=10.6,10.6 \mathrm{~Hz}, 1 \mathrm{H}), 3.96-3.90(\mathrm{~m}, 3 \mathrm{H}), 3.62(\mathrm{~m}, 1 \mathrm{H}), 3.15$ (d, $J=13.4 \mathrm{~Hz}, 1 \mathrm{H}), 3.01$ (br, $1 \mathrm{H})$. The enantiomeric ratio of 3 was determined to be 97:3 in favor of the $R$ enantiomer by CSP-HPLC using racemic material as a standard. (Chiralcel OD column; 10\% 2-propanol in hexane; $0.5 \mathrm{~mL} / \mathrm{min}): 12.7 \mathrm{~min}(R), 19.4 \mathrm{~min}(S)$.

(2-Methoxy-2-oxo-(S)-1-phenylethyl) (R)- $\alpha$-dibenzylamino-propanoate $11 .{ }^{1} \mathrm{H} \mathrm{NMR}\left(\mathrm{CDCl}_{3}\right.$, $400 \mathrm{MHz}) 7.51-7.21(\mathrm{~m}, 15 \mathrm{H}), 5.97$ (s, 3H), 3.89 (d, $J=14.0 \mathrm{~Hz}, 2 \mathrm{H}), 3.80$ (d, J=14.0 Hz, 2H), $3.74(\mathrm{~s}, 3 \mathrm{H}), 3.65(\mathrm{q}, J=7.1 \mathrm{~Hz}, 1 \mathrm{H}), 1.35(\mathrm{~d}, J=7.1 \mathrm{~Hz}, 3 \mathrm{H}) ;{ }^{13} \mathrm{C} \mathrm{NMR}\left(\mathrm{CDCl}_{3}, 100 \mathrm{MHz}\right)$ 173.6, 169.8, 140.4, 134.4, 129.7, 129.3, 129.2, 128.7, 128.1, 127.4, 74.9, 56.3, 54.7, 53.1, 15.5; Anal. calcd for $\mathrm{C}_{26} \mathrm{H}_{27} \mathrm{NO}_{4}$ : C, 74.80; H, 6.52; N, 3.35; Found: C, 74.86; H, 6.67; N, 3.15.

(2-Isopropoxy-2-oxo-(S)-1-phenylethyl) $(\boldsymbol{R})$ - $\alpha$-dibenzylamino-propanoate $12 .{ }^{1} \mathrm{H} \quad \mathrm{NMR}$ $\left(\mathrm{CDCl}_{3}, 400 \mathrm{MHz}\right) 7.51-7.22(\mathrm{~m}, 15 \mathrm{H}), 5.90(\mathrm{~s}, 1 \mathrm{H}), 5.09(\mathrm{~m}, 1 \mathrm{H}), 3.89(\mathrm{~d}, J=14.0 \mathrm{~Hz}, 2 \mathrm{H})$, $3.79(\mathrm{~d}, J=14.0 \mathrm{~Hz}, 2 \mathrm{H}), 3.64(\mathrm{q}, J=7.1 \mathrm{~Hz}, 1 \mathrm{H}), 1.36$ (d, $J=7.0 \mathrm{~Hz}, 3 \mathrm{H}), 1.29$ (d, $J=6.2 \mathrm{~Hz}$, $3 \mathrm{H}), 1.15(\mathrm{~d}, J=6.3 \mathrm{~Hz}, 3 \mathrm{H}) ;{ }^{13} \mathrm{C} \mathrm{NMR}\left(\mathrm{CDCl}_{3}, 100 \mathrm{MHz}\right) 173.6,168.8,140.3,134.5,129.2$, 129.1, 129.0, 128.6, 128.0, 127.3, 75.3, 69.9, 56.3, 54.6, 22.1, 21.9, 15.6; HRMS calcd for $\mathrm{C}_{28} \mathrm{H}_{32} \mathrm{NO}_{4}\left(\mathrm{M}^{+}+1\right): 446.2331$. Found: 446.2332 .

(2-Benzylamino-2-oxo-( $S)$-1-phenylethyl) (R)- $\alpha$-dibenzylamino-propanoate $13 .{ }^{1} \mathrm{H}$ NMR $\left(\mathrm{CDCl}_{3}, 400 \mathrm{MHz}\right) 7.37-7.21(\mathrm{~m}, 20 \mathrm{H}), 6.43(\mathrm{br}, 1 \mathrm{H}), 6.15(\mathrm{~s}, 1 \mathrm{H}), 4.45(\mathrm{~m}, 2 \mathrm{H}), 3.77(\mathrm{~d}, J=$ $14.1 \mathrm{~Hz}, 2 \mathrm{H}), 3.69$ (d, $J=14.1 \mathrm{~Hz}, 2 \mathrm{H}), 3.60(\mathrm{~m}, 1 \mathrm{H}), 1.32(\mathrm{~d}, J=7.0 \mathrm{~Hz}, 3 \mathrm{H}) ;{ }^{13} \mathrm{C}$ NMR $\left(\mathrm{CDCl}_{3}, 100 \mathrm{MHz}\right) 172.4,168.7,139.9,138.1,136.0,129.4,129.2,128.9,128.8,128.1,128.0$, 
127.7, 127.5, 75.9, 56.6, 54.8, 43.7, 14.1; Anal. calcd for $\mathrm{C}_{32} \mathrm{H}_{32} \mathrm{~N}_{2} \mathrm{O}_{3}: \mathrm{C}, 78.02 ; \mathrm{H}, 6.55 ; \mathrm{N}$, 5.69; Found: C, 78.03; H, 6.62; N, 5.77.

(2-Oxo-2-(1-pyrrolidinyl)-(S)-1-phenylethyl) (R)- $\alpha$-dibenzylamino-propanoate $14 .{ }^{1} \mathrm{H}$ NMR $\left(\mathrm{CDCl}_{3}, 400 \mathrm{MHz}\right) 7.54-7.15(\mathrm{~m}, 15 \mathrm{H}), 6.04(\mathrm{~s}, 1 \mathrm{H}), 3.87(\mathrm{~s}, 4 \mathrm{H}), 3.66-3.58(\mathrm{~m}, 3 \mathrm{H}), 3.44-3.41$ $(\mathrm{m}, 1 \mathrm{H}), 3.19-3.13(\mathrm{~m}, 1 \mathrm{H}), 1.92-1.68(\mathrm{~m}, 4 \mathrm{H}), 1.33(\mathrm{~d}, J=7.0 \mathrm{~Hz}, 3 \mathrm{H}) ;{ }^{13} \mathrm{C} \mathrm{NMR}\left(\mathrm{CDCl}_{3}, 100\right.$ MHz) 173.9, 166.8, 140.6, 134.5, 129.4, 129.3, 129.2, 129.1, 128.6, 127.2, 75.0, 56.2, 54.6, 46.8, 46.4, 26.6, 24.3, 15.9; Anal. calcd for $\mathrm{C}_{29} \mathrm{H}_{32} \mathrm{~N}_{2} \mathrm{O}_{3}$ : C, 76.29; H, 7.06; N, 6.14; Found: C, 76.24; H, 7.06; N, 6.13 .

(2-Isopropoxy-2-oxo-(S)-1-phenylethyl) (R)- $\alpha$-dibenzylamino-phenylacetate $15 .{ }^{1} \mathrm{H}$ NMR $\left(\mathrm{CDCl}_{3}, 400 \mathrm{MHz}\right)$ 7.44-7.23 (m, 20H), $5.97(\mathrm{~s}, 1 \mathrm{H}), 5.13(\mathrm{~m}, 1 \mathrm{H}), 4.79(\mathrm{~s}, 1 \mathrm{H}), 3.82(\mathrm{~s}, 4 \mathrm{H})$,

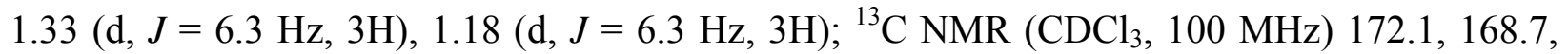
$140.0,136.8,134.2,129.5,129.4,129.2,129.1,128.7,128.6,128.2,128.0,127.4,75.5,70.0$, 65.8, 54.4, 22.2, 21.9; HRMS calcd for $\mathrm{C}_{33} \mathrm{H}_{34} \mathrm{NO}_{4}\left(\mathrm{M}^{+}+1\right)$ : 508.2488. Found: 508.2489.

(2-Benzylamino-2-oxo-(S)-1-phenylethyl) (R)- $\alpha$-dibenzylamino-phenylacetate $16 .{ }^{1} \mathrm{H}$ NMR $\left(\mathrm{CDCl}_{3}, 400 \mathrm{MHz}\right) 7.41-7.16(\mathrm{~m}, 25 \mathrm{H}), 6.36(\mathrm{br}, 1 \mathrm{H}), 6.29(\mathrm{~s}, 1 \mathrm{H}), 4.70(\mathrm{~s}, 1 \mathrm{H}), 4.40(\mathrm{~m}, 2 \mathrm{H})$, $3.74(\mathrm{~m}, 4 \mathrm{H}) ;{ }^{13} \mathrm{C} \mathrm{NMR}\left(\mathrm{CDCl}_{3}, 100 \mathrm{MHz}\right)$ 170.7, 168.5, 139.6, 138.1, 136.2, 135.8, 129.2, $129.1,129.0,128.8,128.7,128.1,128.0,127.5,76.0,66.3,54.5,43.7$; Anal. calcd for $\mathrm{C}_{37} \mathrm{H}_{34} \mathrm{~N}_{2} \mathrm{O}_{3}$ : C, 80.12; H, 6.18; N, 5.05; Found: C, 80.10; H, 6.08; N, 5.01.

(2-(1-Pyrrolidinyl)-2-oxo-(S)-1-phenylethyl) $\quad(R)$ - $\alpha$-dibenzylamino-phenylacetate 17. ${ }^{1} \mathrm{H} \mathrm{NMR}\left(\mathrm{CDCl}_{3}, 400 \mathrm{MHz}\right) 7.48-7.18(\mathrm{~m}, 20 \mathrm{H}), 6.12(\mathrm{~s}, 1 \mathrm{H}), 4.74(\mathrm{~s}, 1 \mathrm{H}), 3.87(\mathrm{~d}, J=13.9 \mathrm{~Hz}$, 2H), $3.79(\mathrm{~d}, J=13.9 \mathrm{~Hz}, 2 \mathrm{H}), 3.69(\mathrm{~m}, 2 \mathrm{H}), 3.46(\mathrm{~m}, 1 \mathrm{H}), 3.21(\mathrm{~m}, 1 \mathrm{H}), 1.99-1.77(\mathrm{~m}, 4 \mathrm{H}) ;{ }^{13} \mathrm{C}$ NMR $\left(\mathrm{CDCl}_{3}, 100 \mathrm{MHz}\right)$ 172.5, 166.7, 140.2, 137.1, 134.1, 129.7, 129.5, 129.3, 129.2, 129.1, 128.6, 128.5, 128.1, 127.3, 75.3, 65.6, 54.4, 46.8, 46.4, 26.6, 24.3; HRMS calcd for $\mathrm{C}_{34} \mathrm{H}_{35} \mathrm{~N}_{2} \mathrm{O}_{3}$ $\left(\mathrm{M}^{+}+1\right): 519.2648$. Found: 519.2646 .

(2-Methoxy-2-oxo-(S)-1-phenylethyl) $\quad(\boldsymbol{R})$ - $\alpha$-dibutylamino-phenylacetate $\quad 20 .{ }^{1} \mathrm{H} \quad \mathrm{NMR}$ $\left(\mathrm{CDCl}_{3}, 400 \mathrm{MHz}\right)$ 7.49-7.29 (m, 10H), $5.97(\mathrm{~s}, 1 \mathrm{H}), 4.77(\mathrm{~s}, 1 \mathrm{H}), 3.72(\mathrm{~s}, 3 \mathrm{H}), 2.59(\mathrm{~m}, 4 \mathrm{H})$,

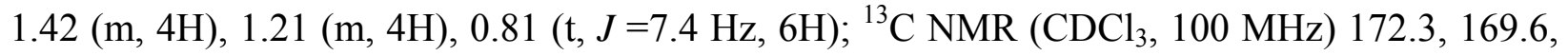
$137.4,134.1,129.5,129.2,129.1,128.6,128.4,128.0,74.9,68.7,52.9,50.7,30.3,20.7,14.4$; Anal. calcd for $\mathrm{C}_{25} \mathrm{H}_{33} \mathrm{NO}_{4}$ : C, 72.96; H, 8.08; N, 3.40. Found: C, 73.01; H, 7.72; N, 3.41.

(2-Methoxy-2-oxo-(S)-1-phenylethyl) (R)- $\alpha$-dibutylamino-propanoate $21 .{ }^{1} \mathrm{H} \mathrm{NMR}\left(\mathrm{CDCl}_{3}\right.$, $400 \mathrm{MHz}$ ) 7.48-7.26 (m, 5H), $5.91(\mathrm{~s}, 1 \mathrm{H}), 3.71$ (s, 3H), 3.67 (q, $J=7.0 \mathrm{~Hz}, 1 \mathrm{H}), 2.64-2.58$ (m, $4 \mathrm{H}), 1.43-1.26(\mathrm{~m}, 11 \mathrm{H}), 0.88(\mathrm{~m}, 6 \mathrm{H}) ;{ }^{13} \mathrm{C} \mathrm{NMR}\left(\mathrm{CDCl}_{3}, 100 \mathrm{MHz}\right) 174.0,169.8,134.4,129.5$, 129.1, 128.0, 74.7, 58.3, 53.0, 51.3, 31.6, 20.8, 16.2, 14.5; Anal. calcd for $\mathrm{C}_{20} \mathrm{H}_{31} \mathrm{NO}_{4}$ : C, 68.74; H, 8.94; N, 4.01; Found: C, 68.70; H, 6.52; N, 3.35 .

(2-Methoxy-2-oxo-(S)-1-phenylethyl) (R)- $\alpha$-diallylamino-phenylacetate $22 .{ }^{1} \mathrm{H} \mathrm{NMR}\left(\mathrm{CDCl}_{3}\right.$, $400 \mathrm{MHz}) 7.42-7.25(\mathrm{~m}, 10 \mathrm{H}), 5.97(\mathrm{~s}, 1 \mathrm{H}), 5.83(\mathrm{~m}, 2 \mathrm{H}), 5.25$ (d, J=17.0 Hz, 2H), 5.14 (d, J= $10.1 \mathrm{~Hz}, 2 \mathrm{H}), 4.85(\mathrm{~s}, 1 \mathrm{H}), 3.71(\mathrm{~s}, 3 \mathrm{H}), 3.29$ (d, $J=6.7 \mathrm{~Hz}, 4 \mathrm{H}) ;{ }^{13} \mathrm{C} \mathrm{NMR}\left(\mathrm{CDCl}_{3}, 100 \mathrm{MHz}\right)$ 172.2 , 169.6, 136.8, 136.1, 134.0, 129.6, 129.2, 129.1, 128.8, 128.4, 128.0, 118.2, 75.1, 67.4, 53.3, 53.0; Anal. calcd for $\mathrm{C}_{23} \mathrm{H}_{25} \mathrm{NO}_{4}$ : C, 72.80; H, 6.64; N, 3.69. Found: C, 73.21; H, 6.38; N, 3.62 . 
(2-Methoxy-2-oxo-(S)-1-phenylethyl) (R)- $\alpha$-diallylamino-propanoate $23 .{ }^{1} \mathrm{H} \mathrm{NMR}\left(\mathrm{CDCl}_{3}\right.$, $400 \mathrm{MHz}) 7.49-7.38(\mathrm{~m}, 5 \mathrm{H}), 5.92(\mathrm{~s}, 1 \mathrm{H}), 5.80(\mathrm{~m}, 2 \mathrm{H}), 5.26(\mathrm{dd}, J=17.1$ and $1.3 \mathrm{~Hz}, 2 \mathrm{H})$, $5.12(\mathrm{~d}, J=10.2 \mathrm{~Hz}, 2 \mathrm{H}), 3.78(\mathrm{q}, J=7.2 \mathrm{~Hz}, 1 \mathrm{H}), 3.73(\mathrm{~s}, 3 \mathrm{H}), 3.36(\mathrm{dd}, J=14.5$ and $5.2 \mathrm{~Hz}$, 2H), $3.24(\mathrm{~d}, J=14.5$ and $7.2 \mathrm{~Hz}, 2 \mathrm{H}), 1.31(\mathrm{~d}, J=7.1 \mathrm{~Hz}, 3 \mathrm{H}) ;{ }^{13} \mathrm{C} \mathrm{NMR}\left(\mathrm{CDCl}_{3}, 100 \mathrm{MHz}\right)$ $173.7,169.7,137.1,134.3,129.6,129.2,128.0,117.5,74.8,57.3,53.8,53.0,15.7$; Anal. calcd for $\mathrm{C}_{18} \mathrm{H}_{23} \mathrm{NO}_{4}$ : C, 68.12; H, 7.30; N, 4.41; Found: C, 68.18; H, 7.32; N, 4.16.

(2-Methoxy-2-oxo-(S)-1-phenylethyl) (R)- $\alpha$-(1-piperidinyl)-propanoate $24 .{ }^{1} \mathrm{H} \mathrm{NMR}\left(\mathrm{CDCl}_{3}\right.$, $400 \mathrm{MHz}) 7.49-7.38(\mathrm{~m}, 5 \mathrm{H}), 5.93(\mathrm{~s}, 1 \mathrm{H}), 3.72(\mathrm{~s}, 3 \mathrm{H}), 3.46(\mathrm{q}, J=7.1 \mathrm{~Hz}, 1 \mathrm{H}), 2.73(\mathrm{~m}, 1 \mathrm{H})$, $2.58(\mathrm{~m}, 3 \mathrm{H}), 1.59(\mathrm{~m}, 4 \mathrm{H}), 1.45(\mathrm{~m}, 2 \mathrm{H}), 1.34(\mathrm{~d}, J=7.1 \mathrm{~Hz}, 3 \mathrm{H}) ;{ }^{13} \mathrm{C} \mathrm{NMR}\left(\mathrm{CDCl}_{3}, 100 \mathrm{MHz}\right)$ 173.1, 169.7, 134.3, 129.6, 129.2, 128.0, 74.7, 63.2, 53.0, 50.8, 26.8, 25.0, 15.3; Anal. calcd for $\mathrm{C}_{17} \mathrm{H}_{23} \mathrm{NO}_{4}$ : C, 66.86; H, 7.59; N, 4.59; O, 20.96. Found: C, 66.80; H, 7.74; N, 4.62.

(2-Methoxy-2-oxo-(S)-1-phenylethyl) (R)- $\alpha$-(1-piperidinyl)-butanoate $25 .{ }^{1} \mathrm{H} \mathrm{NMR}\left(\mathrm{CDCl}_{3}\right.$, $400 \mathrm{MHz}) 7.49-7.38(\mathrm{~m}, 5 \mathrm{H}), 5.93(\mathrm{~s}, 1 \mathrm{H}), 3.72(\mathrm{~s}, 3 \mathrm{H}), 3.21(\mathrm{dd}, J=6.3$ and $8.2 \mathrm{~Hz} 1 \mathrm{H}), 2.75$ $(\mathrm{m}, 1 \mathrm{H}), 2.55(\mathrm{~m}, 3 \mathrm{H}), 1.78(\mathrm{~m}, 2 \mathrm{H}), 1.58-1.43(\mathrm{~m}, 6 \mathrm{H}), 0.92(\mathrm{t}, J=7.4 \mathrm{~Hz}, 3 \mathrm{H}) ;{ }^{13} \mathrm{C}$ NMR $\left(\mathrm{CDCl}_{3}, 100 \mathrm{MHz}\right) 172.5,169.7,134.4,129.6,129.2,128.0,74.6,69.9,52.9,50.8,27.0,25.1$, 23.3, 11.1; Anal. calcd for $\mathrm{C}_{18} \mathrm{H}_{25} \mathrm{NO}_{4}$ : C, 67.69; H, 7.89; N, 4.39; Found: C, 67.75; H, 8.11; N, 4.54.

(2-Methoxy-2-oxo-(S)-1-phenylethyl) (R)- $\alpha$-(1-piperidinyl)-hexanoate $26 .{ }^{1} \mathrm{H} \mathrm{NMR}\left(\mathrm{CDCl}_{3}\right.$, $400 \mathrm{MHz})$ 7.49-7.35 (m, 5H), $5.92(\mathrm{~s}, 1 \mathrm{H}), 3,72(\mathrm{~s}, 1 \mathrm{H}), 3.29(\mathrm{~m}, 1 \mathrm{H}), 2.77(\mathrm{~m}, 1 \mathrm{H}), 2.63(\mathrm{~m}$, $1 \mathrm{H}), 2.54(\mathrm{~m}, 2 \mathrm{H}), 1.85-1.29(\mathrm{~m}, 10 \mathrm{H}), 0.88(\mathrm{t}, J=6.9 \mathrm{~Hz}, 3 \mathrm{H}) ;{ }^{13} \mathrm{C} \mathrm{NMR}\left(\mathrm{CDCl}_{3}, 100 \mathrm{MHz}\right)$ 172.5, 169.7, 134.3, 129.6, 129.2, 128.0, 74.6, 68.3, 52.9, 50.7, 29.7, 28.7, 27.0, 25.1, 23.0, 14.4; Anal. calcd for $\mathrm{C}_{20} \mathrm{H}_{29} \mathrm{NO}_{4}$ : C, 69.14; H, 8.41; N, 4.03; Found C, 69.10; H, 8.58; N, 4.02.

(2-Methoxy-2-oxo-(S)-1-phenylethyl)

(R)- $\alpha$-(3,4-dihydro-2(1H)-isoquinolinyl)-phenyl acetate 27. ${ }^{1} \mathrm{H} \mathrm{NMR}\left(\mathrm{CDCl}_{3}, 400 \mathrm{MHz}\right)$ 7.52-7.07 (m, 14H), $5.93(\mathrm{~s}, 1 \mathrm{H}), 4.44(\mathrm{~s}, 1 \mathrm{H}), 3.75$ (s, 2H), 3.69 (s, 3H), $2.87(\mathrm{~m}, 4 \mathrm{H}) ;{ }^{13} \mathrm{C} \mathrm{NMR}\left(\mathrm{CDCl}_{3}, 100 \mathrm{MHz}\right) 171.6,169.5,136.1,134.9,133.1$, 129.6, 129.4, 129.2, 129.1, 129.0, 128.9, 128.0, 127.2, 126.6, 126.1, 75.2, 73.2, 54.1, 53.1, 48.7, 29.4; Anal. calcd for $\mathrm{C}_{26} \mathrm{H}_{25} \mathrm{NO}_{4}$ : C, 75.16; H, 6.06; N, 3.37; Found: C, 75.03; H, 6.12 N, 3.15.

(2-Methoxy-2-oxo-(S)-1-phenylethyl) $\quad(R)-\alpha$-(3,4-dihydro-2(1H)-isoquinolinyl)-propanoate 28. ${ }^{1} \mathrm{H}$ NMR $\left(\mathrm{CDCl}_{3}, 400 \mathrm{MHz}\right)$ 7.49-7.00 (m, 9H), $5.95(\mathrm{~s}, 1 \mathrm{H}), 3.99(\mathrm{~d}, J=14.6 \mathrm{~Hz}, 1 \mathrm{H}), 3.90$ $(\mathrm{d}, J=14.6 \mathrm{~Hz}, 1 \mathrm{H}), 3.72(\mathrm{~m}, 1 \mathrm{H}), 3.70(\mathrm{~s}, 1 \mathrm{H}), 3.12(\mathrm{~m}, 1 \mathrm{H}), 2.91(\mathrm{~m}, 3 \mathrm{H}), 1.45(\mathrm{~d}, J=7.0 \mathrm{~Hz}$, $3 \mathrm{H}) ;{ }^{13} \mathrm{C} \mathrm{NMR}\left(\mathrm{CDCl}_{3}, 100 \mathrm{MHz}\right) 172.9,169.6,135.4,134.8,134.1,129.7,129.3,129.1,128.1$, 127.0, 126.4, 125.9, 74.9, 62.2, 53.0, 52.3, 47.4, 30.2, 15.6; Anal. calcd for $\mathrm{C}_{21} \mathrm{H}_{23} \mathrm{NO}_{4}$ : C, 71.37; H, 6.56; N, 3.96; Found: C, 71.48; H, 6.59; N, 3.85 .

(2-Methoxy-2-oxo-(S)-1-phenylethyl) $\quad(R)$ - $\alpha$-diphenylmethylamino-phenylacetate $29 .{ }^{1} \mathrm{H}$ NMR $\left(\mathrm{CDCl}_{3}, 400 \mathrm{MHz}\right)$ 7.50-7.23 (m, 20H), 5.96 (s, 1H), 4.89 (s, 1H), 4.51 (s, 1H), 3.75 (s, $1 \mathrm{H}) ;{ }^{13} \mathrm{C} \mathrm{NMR}\left(\mathrm{CDCl}_{3}, 100 \mathrm{MHz}\right) 173.3,169.4,143.8,143.3,138.0,133.9,129.2,129.1,129.0$, 128.5, 128.1, 128.0, 127.9, 127.7, 75.3, 65.0, 63.2, 53.1; Anal. calcd for $\mathrm{C}_{30} \mathrm{H}_{27} \mathrm{NO}_{4}$ : C, 77.40; $\mathrm{H}$, 5.85; N, 3.01;. Found: C, 77.42; H, 6.07; N, 3.00.

(2-Methoxy-2-oxo-(S)-1-phenylethyl) (R)- $\alpha$-diphenylmethylamino-propanoate $30 .{ }^{1} \mathrm{H}$ NMR $\left(\mathrm{CDCl}_{3}, 400 \mathrm{MHz}\right) 7.51-7.18(\mathrm{~m}, 15 \mathrm{H}), 5.97(\mathrm{~s}, 1 \mathrm{H}), 4.95(\mathrm{~s}, 1 \mathrm{H}), 3.77(\mathrm{~s}, 3 \mathrm{H}), 3.46(\mathrm{q}, J=7.0$ 
$\mathrm{Hz}, 1 \mathrm{H}), 2.17$ (br, 1H), $1.34(\mathrm{~d}, J=7.1 \mathrm{~Hz}, 3 \mathrm{H}) ;{ }^{13} \mathrm{C} \mathrm{NMR}\left(\mathrm{CDCl}_{3}, 100 \mathrm{MHz}\right) 170.1,169.5$, 144.6, 134.1, 129.7, 129.2, 128.9, 128.1, 127.8, 127.7, 75.0, 65.5, 54.8, 53.1, 19.8; HRMS calcd for $\mathrm{C}_{25} \mathrm{H}_{26} \mathrm{NO}_{4}\left(\mathrm{M}^{+}+1\right)$ : 404.1862 . Found: 404.1861 .

(2-Methoxy-2-oxo-(S)-1-phenylethyl) (R)- $\alpha$-benzylamino-phenylacetate $31 .{ }^{1} \mathrm{H} \mathrm{NMR}\left(\mathrm{CDCl}_{3}\right.$, $400 \mathrm{MHz})$ 7.47-7.21 (m, 15H), $5.94(\mathrm{~s}, 1 \mathrm{H}), 4.59(\mathrm{~s}, 1 \mathrm{H}), 3.80(\mathrm{~m}, 2 \mathrm{H}), 3.70(\mathrm{~s}, 3 \mathrm{H}), 2.30$ (br, $1 \mathrm{H}) ;{ }^{13} \mathrm{C} \mathrm{NMR}\left(\mathrm{CDCl}_{3}, 100 \mathrm{MHz}\right) 173.1,169.5,139.9,137.9,133.9,129.6,129.1,128.9,128.8$, 128.6, 128.0, 127.8, 127.6, 75.3, 64.7, 53.1, 51.8; Anal. calcd for $\mathrm{C}_{24} \mathrm{H}_{23} \mathrm{NO}_{4}$ : C, 74.02; H, 5.95; N, 3.60;. Found: C, 74.04; H, 5.93 N, 3.64.

(2-Methoxy-2-oxo-(S)-1-phenylethyl) (R)- $\alpha$-benzylamino-butanoate $32 .{ }^{1} \mathrm{H} \mathrm{NMR}\left(\mathrm{CDCl}_{3}\right.$, $400 \mathrm{MHz}$, major diastereomer) 7.47-7.25 (s, 10H), $5.98(\mathrm{~s}, 1 \mathrm{H}), 3.87(\mathrm{~d}, J=12.9 \mathrm{~Hz}, 1 \mathrm{H}), 3.75$ (s, 3H), $3.67(\mathrm{~d}, J=12.9 \mathrm{~Hz}, 1 \mathrm{H}), 3.36(\mathrm{~m}, 1 \mathrm{H}), 1.73(\mathrm{~m}, 2 \mathrm{H}), 0.93(\mathrm{t}, J=7.4 \mathrm{~Hz}, 3 \mathrm{H})$. The spectral data of 32 were identical to those of the authentic material reported previously. ${ }^{\mathrm{e}}$

$\boldsymbol{N}$-Benzyl-3-(R)-phenyl-morpholin-2-one 33. ${ }^{1} \mathrm{H}$ NMR $\left(\mathrm{CDCl}_{3}, 400 \mathrm{MHz}\right)$ 7.58-7.24 (m, 10H), 4.55 (dt, $J=11.0 \mathrm{~Hz}, 3.1 \mathrm{~Hz}, 1 \mathrm{H}), 4.37$ (m, 1H), 4.26 (s, 1H), 3.77 (d, $J=13.4 \mathrm{~Hz}, 1 \mathrm{H}), 3.17$ (d, $J=13.3 \mathrm{~Hz}, 1 \mathrm{H}), 2.99(\mathrm{~m}, 1 \mathrm{H}), 2.64(\mathrm{~m}, 1 \mathrm{H})$. The spectral data of 33 were identical to those of the authentic material reported previously. ${ }^{6 a}$ CSP-HPLC (Chiralpak AD-H column; 10\% 2propanol in hexane; $0.5 \mathrm{~mL} / \mathrm{min}$ ) 90:10 er, $20.9 \mathrm{~min}$ (major enantiomer), $24.7 \mathrm{~min}$ (minor enantiomer).

N-Benzyl-3-(R)-methyl-morpholin-2-one 34. ${ }^{1} \mathrm{H}$ NMR $\left(\mathrm{CDCl}_{3}, 400 \mathrm{MHz}\right)$ 7.34-7.28 (m, 5H), $4.32(\mathrm{~m}, 2 \mathrm{H}), 3.96(\mathrm{~d}, J=13.4 \mathrm{~Hz}, 1 \mathrm{H}), 3.42$ (q, $J=6.9 \mathrm{~Hz}, 1 \mathrm{H}), 3.33(\mathrm{~d}, J=13.4 \mathrm{~Hz}, 1 \mathrm{H}), 2.86$ $(\mathrm{dt}, J=12.9 \mathrm{~Hz}, 3.7 \mathrm{~Hz}, 1 \mathrm{H}), 2.52(\mathrm{~m}, 1 \mathrm{H}), 1.56(\mathrm{~d}, J=6.7 \mathrm{~Hz}, 3 \mathrm{H})$. The spectral data of 35 were identical to those of the authentic material reported previously. ${ }^{7}$ CSP-HPLC (Chiralpak OJ$\mathrm{H}$ column; 5\% 2-propanol in hexane; $0.5 \mathrm{~mL} / \mathrm{min}$ ) 84:16 er, $54.6 \mathrm{~min}$ (major enantiomer), 56.4 min (minor enantiomer).

$\boldsymbol{N}$-Benzyl-3-(R)-butyl-morpholin-2-one 35. ${ }^{1} \mathrm{H}$ NMR $\left(\mathrm{CDCl}_{3}, 400 \mathrm{MHz}\right)$ 7.37-7.26 (m, 5H), 4.35-4.26 (m, 1H), $3.97(\mathrm{~d}, \mathrm{~J}=13.4 \mathrm{~Hz}, 1 \mathrm{H}), 3.36$ (t, $J=4.5 \mathrm{~Hz}, 1 \mathrm{H}), 3.29$ (d, J=13.4 Hz, 1H), $2.88(\mathrm{~m}, 1 \mathrm{H}), 2.50(\mathrm{~m}, 1 \mathrm{H}), 2.09-1.86(\mathrm{~m}, 2 \mathrm{H}), 1.62(\mathrm{~m}, 1 \mathrm{H}), 1.36-1.31(\mathrm{~m}, 3 \mathrm{H}), 0.92(\mathrm{t}, \mathrm{J}=7.0$ $\mathrm{Hz}, 3 \mathrm{H}) ;{ }^{13} \mathrm{C} \mathrm{NMR}\left(\mathrm{CDCl}_{3}, 100 \mathrm{MHz}\right) 171.4,137.8,129.2,129.0,127.9,68.2,65.3,59.1,47.1$, 30.9, 27.6, 23.1, 14.4; Anal. calcd for $\mathrm{C}_{15} \mathrm{H}_{21} \mathrm{NO}_{2}$ : C, 72.84; $\mathrm{H}, 8.56$; N, 5.66; Found: C, 72.77; H, 8.67; N, 5.73; CSP-HPLC (Chiralpak OD column; 5\% 2-propanol in hexane; $0.5 \mathrm{~mL} / \mathrm{min}$ ) 68:32 er, $22.2 \mathrm{~min}$ (minor enantiomer), $28.2 \mathrm{~min}$ (major enantiomer).

$\boldsymbol{N}$-(m-Methylbenzyl)-3-(R)-phenyl-morpholin-2-one $36 .{ }^{1} \mathrm{H} \mathrm{NMR}\left(\mathrm{CDCl}_{3}, 400 \mathrm{MHz}\right) 7.58$ $7.03(\mathrm{~m}, 9 \mathrm{H}), 4.54(\mathrm{dt}, J=11.4 \mathrm{~Hz}, 3.0 \mathrm{~Hz}, 1 \mathrm{H}), 4.34(\mathrm{~m}, 1 \mathrm{H}), 4.24(\mathrm{~s}, 1 \mathrm{H}), 3.73(\mathrm{~d}, J=13.3 \mathrm{~Hz}$, $1 \mathrm{H}), 3.12(\mathrm{~d}, J=13.3 \mathrm{~Hz}, 1 \mathrm{H}), 2.97(\mathrm{~m}, 1 \mathrm{H}), 2.62(\mathrm{~m}, 1 \mathrm{H}), 2.32(\mathrm{~s}, 3 \mathrm{H}) ;{ }^{13} \mathrm{C} \mathrm{NMR}\left(\mathrm{CDCl}_{3}, 100\right.$ MHz) 169.3, 138.5, 137.9, 137.2, 130.0, 129.3, 129.2, 128.9, 128.8, 128.7, 126.3, 71.0, 69.1, 59.2, 47.3, 21.8; HRMS calcd for $\mathrm{C}_{18} \mathrm{H}_{20} \mathrm{NO}_{2}\left(\mathrm{M}^{+}+1\right)$ : 282.1494. Found: 282.1497; CSP-HPLC (Chiralpak AD-H column; 10\% 2-propanol in hexane; $0.5 \mathrm{~mL} / \mathrm{min}$ ) $81: 19$ er, $17.0 \mathrm{~min}$ (major enantiomer), 19.8min (minor enantiomer).

$\boldsymbol{N}$-(p-Methoxybenzyl)-3-(R)-phenyl-morpholin-2-one 37. ${ }^{1} \mathrm{H} \mathrm{NMR}\left(\mathrm{CDCl}_{3}, 400 \mathrm{MHz}\right) 7.58$ $7.31(\mathrm{~m}, 5 \mathrm{H}), 7.16(\mathrm{~d}, J=8.5 \mathrm{~Hz}, 2 \mathrm{H}), 6.85(\mathrm{~d}, J=8.5 \mathrm{~Hz}, 2 \mathrm{H}), 4.54(\mathrm{dt}, J=11.0 \mathrm{~Hz}, 3.0 \mathrm{~Hz}$, 
$1 \mathrm{H}), 4.37(\mathrm{~m}, 1 \mathrm{H}), 4.24(\mathrm{~s}, 1 \mathrm{H}), 3.79(\mathrm{~s}, 3 \mathrm{H}), 3.71(\mathrm{~d}, J=13.2 \mathrm{~Hz}, 1 \mathrm{H}), 3.12(\mathrm{~d}, J=13.2 \mathrm{~Hz}$, 1H), 2.99 (m, 1H), $2.64(\mathrm{~m}, 1 \mathrm{H}) ;{ }^{13} \mathrm{C} \mathrm{NMR}\left(\mathrm{CDCl}_{3}, 100 \mathrm{MHz}\right) 169.3,159.5,138.0,130.5$, 129.3, 129.2, 129.1, 128.8, 114.2, 70.8, 69.1, 58.6, 55.7, 47.1; HRMS calcd for $\mathrm{C}_{18} \mathrm{H}_{20} \mathrm{NO}_{3}$ $\left(\mathrm{M}^{+}+1\right)$ : 298.1443. Found: 298.1445; CSP-HPLC (Chiralpak AD-H column; 10\% 2-propanol in hexane; 0.5mL/min) 91:9 er, $27.1 \mathrm{~min}$ (major enantiomer), $33.7 \mathrm{~min}$ (minor enantiomer).

$\boldsymbol{N}$-(1-Naphtylmethyl)-3-(S)-phenyl-morpholin-2-one 38. ${ }^{1} \mathrm{H}$ NMR $\left(\mathrm{CDCl}_{3}, 400 \mathrm{MHz}\right) 7.82$ $7.38(\mathrm{~m}, 12 \mathrm{H}), 4.43(\mathrm{dt}, J=10.9 \mathrm{~Hz}, 2.6 \mathrm{~Hz}, 1 \mathrm{H}), 4.29(\mathrm{~s}, 1 \mathrm{H}), 4.28(\mathrm{~m}, 1 \mathrm{H}), 4.19(\mathrm{~d}, J=13.0$ $\mathrm{Hz}, 1 \mathrm{H}), 3.51(\mathrm{~d}, J=13.0 \mathrm{~Hz}, 1 \mathrm{H}), 2.89(\mathrm{~m}, 1 \mathrm{H}), 2.62(\mathrm{~m}, 1 \mathrm{H}) ;{ }^{13} \mathrm{C} \mathrm{NMR}\left(\mathrm{CDCl}_{3}, 100 \mathrm{MHz}\right)$ $169.4,138.0,134.3,133.0,132.6,129.7,129.2$, 129.1, 129.0, 128.9, 128.3, 126.3, 126.2, 125.5, 124.9, 72.2, 69.1, 58.0, 47.3; Anal. calcd for $\mathrm{C}_{21} \mathrm{O}_{19} \mathrm{NO}_{2}: \mathrm{C}, 79.47$; $\mathrm{H}, 6.03$; $\mathrm{N}, 4.41$; Found: $\mathrm{C}$, 79.38; H, 6.20; N, 4.41; CSP-HPLC (Chiralpak AD-H column; 10\% 2-propanol in hexane; $0.5 \mathrm{~mL} / \mathrm{min}$ ) $91: 9 \mathrm{er}, 22.6 \mathrm{~min}$ (major enantiomer), $27.8 \mathrm{~min}$ (minor enantiomer).

$\boldsymbol{N}$-(p-Methoxyphenyl)-3-(S)-phenyl-morpholin-2-one 39. ${ }^{1} \mathrm{H} \mathrm{NMR}\left(\mathrm{CDCl}_{3}, 400 \mathrm{MHz}\right) 7.52$ $7.33(\mathrm{~m}, 5 \mathrm{H}), 6.83(\mathrm{~d}, J=9.0 \mathrm{~Hz}, 2 \mathrm{H}), 6.61(\mathrm{~d}, J=9.0 \mathrm{~Hz}, 2 \mathrm{H}), 5.43(\mathrm{~s}, 1 \mathrm{H}), 4.47(\mathrm{~m}, 2 \mathrm{H}), 3.75$ (s, 3H), $3.68(\mathrm{~m}, 2 \mathrm{H}) ;{ }^{13} \mathrm{C} \mathrm{NMR}\left(\mathrm{CDCl}_{3}, 100 \mathrm{MHz}\right)$ 169.0, 153.4, 142.0, 136.9, 129.5, 128.8, 119.8, 115.4, 114.3, 65.4, 65.0, 56.1, 44.4; HRMS calcd for $\mathrm{C}_{17} \mathrm{H}_{17} \mathrm{NO}_{3}\left(\mathrm{M}^{+}\right)$: 283.1208. Found: 283.1208; CSP-HPLC (Chiralpak AD-H column; 10\% 2-propanol in hexane; $0.5 \mathrm{~mL} / \mathrm{min}$ ) 95:5 er, $38.1 \mathrm{~min}$ (major enantiomer), $42.8 \mathrm{~min}$ (minor enantiomer).

$\boldsymbol{N}$-Benzyl-3-(R)-(p-fluorophenyl)-morpholin-2-one 41. ${ }^{1} \mathrm{H} \mathrm{NMR}\left(\mathrm{CDCl}_{3}, 400 \mathrm{MHz}\right)$ 7.58-7.07 $(\mathrm{m}, 9 \mathrm{H}), 4.54(\mathrm{dt}, J=11.1$ and $3.0 \mathrm{~Hz}, 1 \mathrm{H}), 4.37(\mathrm{~m}, 1 \mathrm{H}), 4.24(\mathrm{~s}, 1 \mathrm{H}), 3.77(\mathrm{~d}, J=13.3 \mathrm{~Hz}, 1 \mathrm{H})$, $3.16(\mathrm{~d}, J=13.4 \mathrm{~Hz}, 1 \mathrm{H}), 2.99(\mathrm{dd}, J=12.9$ and $2.1 \mathrm{~Hz}, 1 \mathrm{H}), 2.65(\mathrm{~m}, 1 \mathrm{H})$. The spectral data of 41 were identical to those of the authentic material reported previously. ${ }^{6 c}$ CSP-HPLC (Chiralpak AD-H column; 10\% 2-propanol in hexane; 0.5mL/min) 90:10 er, $20.0 \mathrm{~min}$ (major enantiomer), 23.3 min (minor enantiomer).

$\boldsymbol{N}$-(p-Methoxyphenyl)-3-(S)-(p-fluorophenyl)-morpholin-2-one $42 .{ }^{1} \mathrm{H}$ NMR $\left(\mathrm{CDCl}_{3}, 400\right.$ MHz) 7.49 (dd, $J=5.6$ and $8.5 \mathrm{~Hz}, 2 \mathrm{H}), 7.06(\mathrm{t}, J=8.6 \mathrm{~Hz}, 2 \mathrm{H}), 6.83(\mathrm{~d}, J=9.0 \mathrm{~Hz}, 2 \mathrm{H}), 6.61$ $(\mathrm{d}, J=9.0 \mathrm{~Hz}, 2 \mathrm{H}), 5.36(\mathrm{~s}, 1 \mathrm{H}), 4.49(\mathrm{~m}, 2 \mathrm{H}), 3.75(\mathrm{~s}, 3 \mathrm{H}), 3.65(\mathrm{~m}, 2 \mathrm{H}) ;{ }^{13} \mathrm{C} \mathrm{NMR}\left(\mathrm{CDCl}_{3}\right.$, $100 \mathrm{MHz}) 168.9,163.0(\mathrm{~d}, J=246.2 \mathrm{~Hz}, 2 \mathrm{H}), 153.7,141.8,132.7,128.6(\mathrm{~d}, J=8.4 \mathrm{~Hz}, 2 \mathrm{H})$, $116.5(\mathrm{~d}, J=21.5 \mathrm{~Hz}, 2 \mathrm{H}), 115.4,114.7,65.6,64.7,56.1,44.6$; HRMS calcd for $\mathrm{C}_{17} \mathrm{H}_{16} \mathrm{FNO}_{3}$ $\left(\mathrm{M}^{+}\right)$: 301.1114. Found: 301.1116; CSP-HPLC (Chiralpak AD-H column; 10\% 2-propanol in hexane; $0.5 \mathrm{~mL} / \mathrm{min}$ ) 94:6 er, $41.6 \mathrm{~min}$ (major enantiomer), $43.2 \mathrm{~min}$ (minor enantiomer).

\section{Acknowledgements}

This work was supported by a grant from the Korea Research Foundation (KRF-2009-0089650). 


\section{References}

1. For reviews on pantolactone auxiliary, see: (a) Koh, K.; Ben, R. N.; Durst, T. Tetrahedron Lett. 1993, 34, 4473. (b) Koh, K.; Durst, T. J. Org. Chem. 1994, 59, 4683. (c) Koh, K.; Ben, R. N.; Durst, T. Tetrahedron Lett. 1994, 35, 375. (d) O'Meara, J. A.; Gardee, N.; Jung, M.; Ben, R. N.; Durst, T. J. Org. Chem. 1998, 63, 3117. (e) Ben, R. N.; Durst, T. J. Org. Chem. 1999, 64, 7700 .

2. For reviews on diacetone-D-glucose auxiliary, see: (a) Kim, H. J.; Shin, E.-k.; Chang, J.-y.; Kim, Y.; Park, Y. S. Tetrahedron Lett. 2005, 46, 4115. (b) Kim, H. J.; Kim, Y.; Choi, E. T.; Lee, M. H.; No, E. S.; Park, Y. S. Tetrahedron 2006, 62, 6303. (c) Kim, Y.; Lee, M. H.; Choi, E. T.; No, E. S.; Park, Y. S. Heterocycles 2007, 71, 5. (d) Kang, K. H.; Lee, M. H.; Choi, E. T.; Park, Y. S. Bull. Korean Chem. Soc. 2007, 28, 1199.

3. For reviews on lactamide auxiliary, see: (a) Devine, P. N.; Dolling, U.-H.; Heid, R. M.; Tschaen, D. M. Tetrahedron Lett. 1996, 37, 2683. (b) Devine, P. N.; Heid, R. M.; Tschaen, D. M. Tetrahedron 1997, 53, 6739. (c) Devine, P. N.; Foster, B. S.; Grabowski, E. J. J.; Reider, P. J. Heterocycles 2002, 58, 119. (d) Ammazzalorso, A.; Amoroso, R.; Bettoni, G.; De Filippis, B.; Giampietro, L.; Maccallini, C.; Tricca, M. L. Arkivoc 2004, 375. (e) Ammazzalorso, A.; Amoroso, R.; Bettoni, G.; De Filippis, B.; Fantacuzzi, M.; Giampietro, L.; Maccallini, C.; Tricca, M. L. Eur. J. Org. Chem. 2006, 4088.

4. Lee, Y. M.; Park, Y. S. Heterocycles 2009, 78, 2233.

5. In the reference $3 \mathrm{c}$, Devine et al reported that no spontaneous cyclization was observed in the reactions of lactamide-derived $\alpha$-halo phenylacetates with $N$-benzyl 2-aminoethanol. Also, we found that reactions of $\alpha$-substituted methyl $\alpha$-bromo acetates with $\mathrm{N}$-benzyl 2aminoethanol did not gave the cyclized products.

6. (a) Ashwood, M. S.; Cottrell, I. F.; Davies, A. J. Tetrahedron: Asymmetry 1997, 8, 957. (b) Zhao, M. M.; McNamara, J. M.; Ho, G.-J.; Emerson, K. M.; Song, Z. J.; Tschaen, d. M.; Brands, K. M. J.; Dolling, U.-H.; Grabowski, E. J. J.; Reider, P. J. J. Org. Chem. 2002, 67, 6743. (c) Kolla, N.; Elati, C. R.; Arunagiri, M.; Gangula, S.; Vankawala, P. J.; Anjaneyulu, Y.; Bhattacharya, A.; Venkatraman, S.; Mathad, V. T. Org. Process Res. Dev. 2007, 11, 455. (d) Elati, C. R.; Kolla, N.; Gangula, S.; Naredla, A.; Vankawala, P. J.; Avinigiri, M. L.; Chalamala, S.; Sundaram, V.; Mathad, V. T.; Bhattacharya, A.; Bandichhor, R. Tetrahedron Lett. 2007, 48, 8001.

7. (a) Kashima, C.; Harada, K. J. Chem. Soc., Perkin Trans. 1 1988, 1521. (b) Kashima, C.; Harada, K. J. Org. Chem. 1989, 54, 789. 